\title{
Giant Hydronephrosis in Sourou Sanou University Teaching Hospital of Bobo-Dioulasso (Burkina-Faso). Two Cases Reports and Literature Review
}

\author{
Timothée Kambou1, Adama Ouattara ${ }^{1 *}$, Abdoul Karim Paré1, Drissa S. Barro², \\ Clotaire Yaméogo ${ }^{3}$, Abdoulaye Kodo ${ }^{3}$, Aristide F. Kabore ${ }^{3}$ \\ ${ }^{1}$ Urology Division, Souro Sanou University Teaching Hospital, Bobo-Dioulasso, Burkina Faso \\ ${ }^{2}$ Anesthesiology and Resuscitation Division, Souro Sanou University Teaching Hospital, Bobo-Dioulasso, Burkina Faso \\ ${ }^{3}$ Urology Division, Yalgado Ouedraogo University Teaching Hospital, Ouagadougou, Burkina Faso \\ Email:^adamsouat1@hotmail.com
}

How to cite this paper: Kambou, T., Ouattara, A., Paré, A.K., Barro, D.S., Yaméogo, C., Kodo, A. and Kabore, A.F. (2018) Giant Hydronephrosis in Sourou Sanou University Teaching Hospital of Bobo-Dioulasso (Burkina-Faso). Two Cases Reports and Literature Review. Open Journal of Urology, 8, 17-24.

https://doi.org/10.4236/oju.2018.81003

Received: December 20, 2017

Accepted: January 23, 2018

Published: January 26, 2018

Copyright $\odot 2018$ by authors and Scientific Research Publishing Inc. This work is licensed under the Creative Commons Attribution International License (CC BY 4.0).

http://creativecommons.org/licenses/by/4.0/

\begin{abstract}
Giant Hydronephrosis (GH) is a rare condition in urology literature and defined as a pelvicalyceal system of kidney containing more of $1000 \mathrm{ml}$ of urine. This condition is not so rare in our setting. We herein, reported two cases of giant hydronephrosis, seen in two young patients respectively with 5 and 9 years old and their collecting system containing $4000 \mathrm{ml}$ and $5000 \mathrm{ml}$ of urines respectively. Only simple nephrectomy was performed for the two cases with renal function impairment and the post operative course was uneventful. Our purpose through these cases reports is to discuss diagnosis features and management of such condition in our setting, a context of low income countries as Burkina-Faso where diagnosis tool and further investigation are not always available.
\end{abstract}

\section{Keywords}

Giant Hydronephrosis, Child, Simple Nephrectomy

\section{Introduction}

Giant hydronephrosis (GH) was first defined by Stirling in 1939 arbitrarily as a urine collection of more than $1000 \mathrm{mls}$ in the excretory cavities [1]. It could also be defined as an important dilatation of the pelvicalyceal system occupying a wide space in the abdominal cavity or a dilatation containing more than one litre of urine and crossing the median line on an X-ray [2]. It is a rare condition re- 
sponsible for an important abdominal deformation with impairment of renal function. Giant Hydronephrosis is more common in male patients than female and the main cause is the ureteropelvic junction obstruction. Despite the availability of diagnostic tool like ultrasound (US) and magnetic resonance imaging (MRI) in western countries, where hydronephrosis diagnosis is made in antenatal period or early detected in newborn, in our setting as developing countries, giant hydronephrosis is often discovered in late stage, where simple nephrectomy remains the only solution for it management. The objective of these serial cases was to discuss diagnosis features and management of such condition in our urology division.

\section{Case Report 1}

It was a 5 years old male patient referred from paediatrics department with a left renal mass. She was admitted in paediatrics with history of fever (Temperature was $38.7^{\circ} \mathrm{C}$ ), vomiting, convulsions and distended abdomen responsible of dyspnoea. A first diagnosis of severe malaria was made and the child was treated accordingly with artesunate base derivatives, paracetamol and oxygen. After improvement of the general state of the patient, an abdominal ultrasound scan was done and based on the results the child was transferred to the urology division for further investigation and treatment. History of the disease was progressive abdominal distension for the past 3 years without any other associated symptoms. From history also it was revealed that she was found two years ago in abdominal ultrasound a left polycystic renal mass in another health center but never go for follow up in any urology division. Physical examination revealed a voluminous abdominal distension with a huge mass occupying the left hypochondrium, left flank, umbilical region, epigastric region and crossing to the right hypochondrium and right flank (Figure 1).

The mass was dull in percussion. Further investigations shows good renal function with serum creatinine at $46.56 \mu \mathrm{mol} / \mathrm{L}$, blood sugar at $3.8 \mathrm{mmol} / \mathrm{L}$, moderate
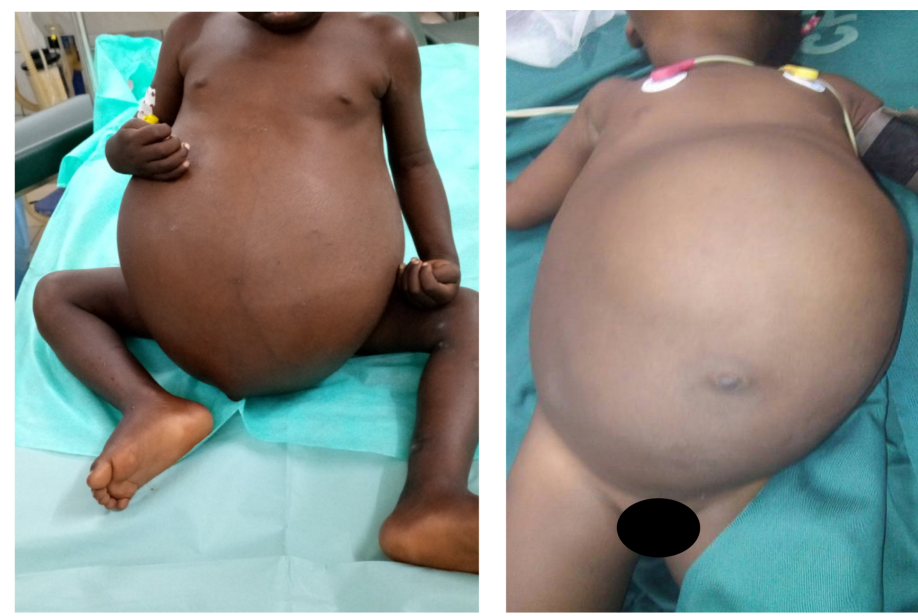

Figure 1. Preoperative image of child with huge abdominal mass occupying the left hypochondrium, left flank, umbilical region. 
anaemia with haemoglobin at $8.7 \mathrm{~g} / \mathrm{dl}$. Urine analysis yielded no bacteria growth. Abdominal ultrasound revealed a voluminous cystic left renal mass with the fluid which contains is estimated at $4000 \mathrm{ml}$, with distorted renal structure and renal parenchyma. Computerizing tomography scan (CT scan) revealed a giant left hydronephrosis destroying the whole renal parenchyma giving a cystic mass of 4000 $\mathrm{ml}$ of urines compatible with a probable complication of ureteropelvic junction syndrome (Figure 2).

A left simple nephrectomy was done under general anesthesia after correction of the anaemia and patient preparation, the whole left kidney was removed in bloc as a cystic mass containing about 4 liters of clear urine of which the culture was sterile (Figure 3). The post-operative course was uneventful with good evolution; any complication has been reported. The child gets better improvement of her general conditions with total recovery. The patient was seen after three months in routine check up with no complain and with a normal serum creatinine at $41.6 \mu \mathrm{mol} / \mathrm{L}$.

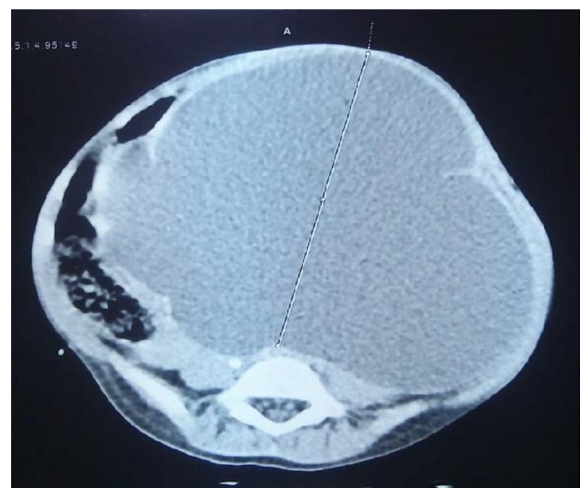

Figure 2. Abdominal CT scan revealed a cystic mass (giant hydronephrosis) with 4000 $\mathrm{ml}$ of urines.

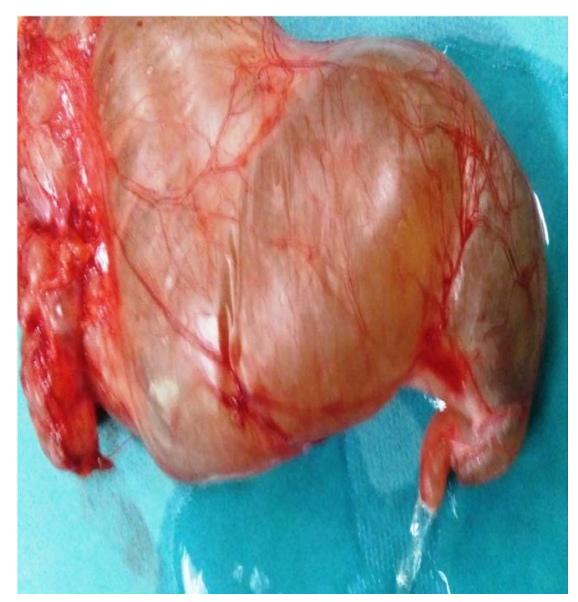

Figure 3. Specimen of renal cystic mass after nephrectomy. The aspect of the specimen is suggestive of ureteropelvic junction obstruction. 


\section{Case Report 2}

It is referred to a 9 years old female, seen in urology consultation with an intermittent painful right lumbar mass. She presented this mass since 8 months but didn't report another associated symptoms. The history not revealed urolithiasis or schistosomiasis in the past nor previous surgical operation in urinary tract. The left flank pain was intermittent with no known aggravating or relieving factors. On physical examination, the abdomen was asymmetrical due to a huge mass in the right hypochondrium region overlapping the median line and reaching the contralateral flank and periumbilical region. The right lumbar contact was present with a dull percussion note (Figure 4).

Blood investigations showed normal serum creatinine at $71 \mathrm{Umol} / 1$, no bacteria growth from urine analysis, normal glycaemia at $3.3 \mathrm{mmol} / \mathrm{l}$ and moderate anaemia with haemoglobin at $11.7 \mathrm{~g} / \mathrm{dl}$. Abdominal ultrasound performed revealed a right voluminous hydronephrosis without any obvious calculus or obstruction within urinary tract. CT scan showed a voluminous pelvicalyceal dilatation crossing the midline and associated with thinning of renal parenchyma. The volume of fluid was estimated at 5 liters hence the diagnosis of right giant hydronephrosis was made. A probable obstruction of ureteropelvic junction was suspected (Figure 5).

After patient preparation and with this non-functioning right kidney, the patient underwent under general anesthesia for simple left nephrectomy. Intraoperatively, a huge cystic mass with ureteropelvic junction obstruction was removed. Renal parenchyma was totally thin and destroyed with a non-dilated right ureter (Figure 6). The postoperative course was simple; any complications were recorded.

\section{Discussion}

Giant hydronephrosis is a rare clinical entity [3] [4]. Since 1910 till 1990, 523

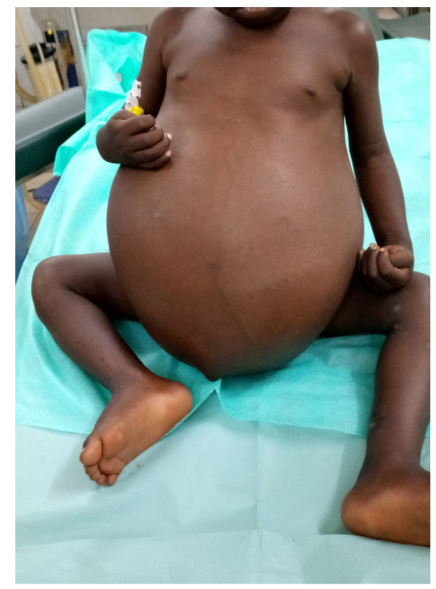

(a)

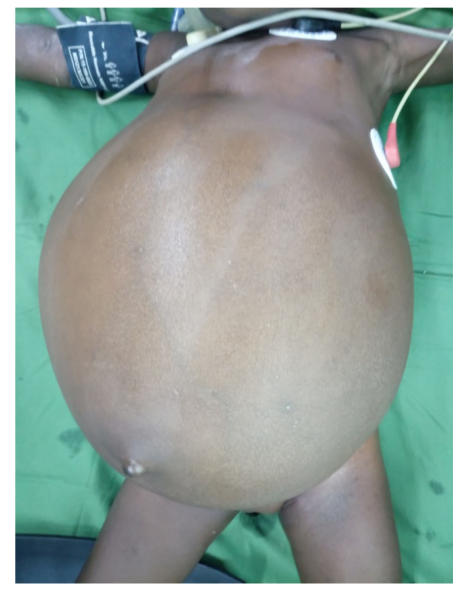

(b)

Figure 4. Preoperative image of child with huge abdominal mass. In sitting position (a), supine position (b). 


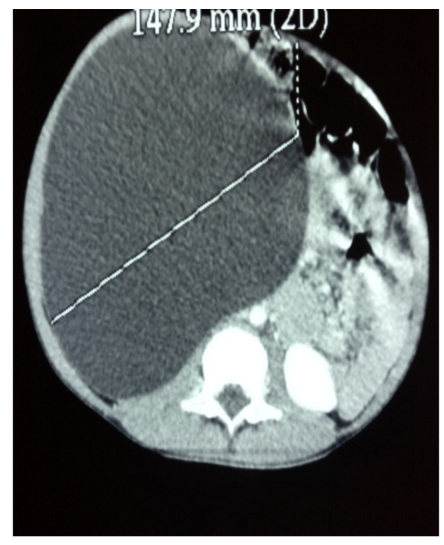

(a)

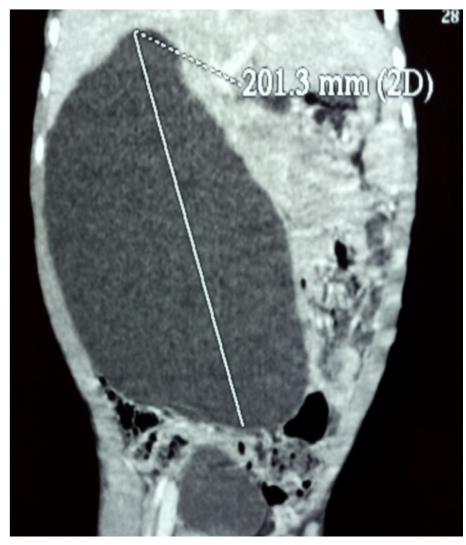

(b)

Figure 5. Axial CT contrast enhanced of abdomen showing a giant hydronephrosis (a). Right kidney destroyed by giant hydronephrosis at CT scan in coronal section (b).

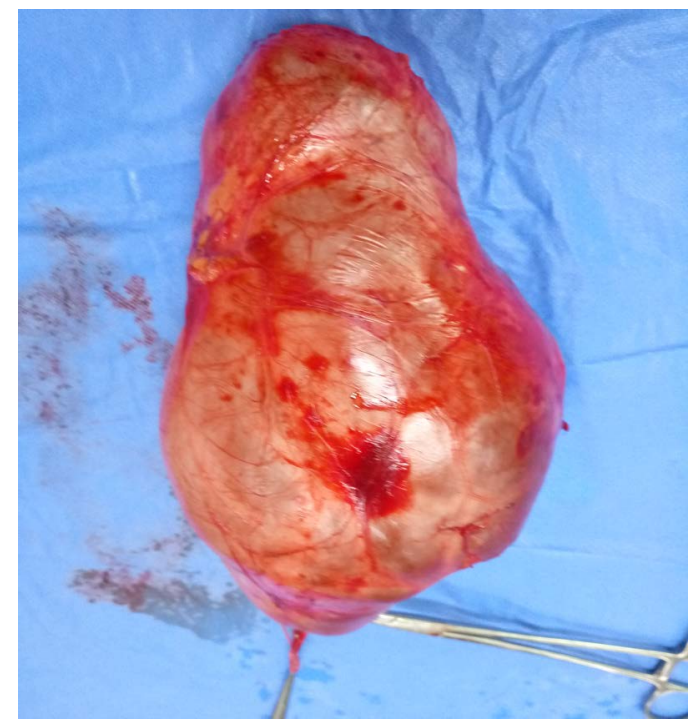

Figure 6. Specimen of giant hydronephrosis after removal. The aspect of the specimen is suggestive of ureteropelvic junction obstruction.

cases of giant hydronephrosis have been reported in the literature [5]. In 1977, Oschner et al. revealed that more than 600 cases were described worldwide [6]. The true incidence rate is difficult to be determined due to its diagnostic errors Diao et al. have reported 7 cases of giant hydronephrosis in 7 years with an average of one case per year in Aristide Dantec Urology division at Dakar in Senegal [7]. Giant hydronephrosis is characterised by an important dilatation of the entire pelvicalyceal system. Sometime complete destruction of the renal parenchyma is occurred. The destruction of the parenchyma by obstruction could be a gradual process but discovered at later age or it could be developed rapidly as observed for our second observation [8]. The initial stage of hydronephrosis is a small collection separated by septae of the dilated calyceal system; it contains 
non concentrated aqueous urine and its walls are formed by the adjoining of the excretory system to the renal capsule. This is shown by the CT scan of patients. The evaluation of the urine volume contained in pelvicalyceal system during giant hydronephrosis varies from $1000 \mathrm{ml}$ of urine to more than $15,000 \mathrm{ml}$ in somes cases reports. Vichwanath et al. described a case report with $15,000 \mathrm{ml}$ of urine [9]. Diao et al. got from their patients a volume of about 2.74 to 6 litres of urine [7]. In our cases, the amount of urine in the collecting system was respectively $4000 \mathrm{ml}$ and $5000 \mathrm{ml}$. According to Benchekroun, some authors reported the associated lesions within giant hydronephrosis; such as renal dysplasia and even focal renal carcinoma [10]. The hypothesis of prolong contact of the renal parenchyma with the urinary metabolites was suggested as the cause of the neoplastic transformation. Kimura et al. have reported a case of giant hydronephrosis associated with sarcomatoid renal cell carcinoma [11]. A case of mucinous cyst adenoma of the upper excretory ways revealed by a giant hydronephrosis was reported by Baka and colleagues [12]. We didn't observe such presentation with associated lesion in our cases. The common clinical manifestation is the voluminous abdominal mass distorting the abdomen. Others associated signs like abdominal or lumbar pain, constipation, dyspnoea and haematuria could be also observed in some cases. Giant hydronephrosis could also present as an occlusive syndrome due to compression of the bowel in rare cases of ectopic kidney [13] [14]. Use of abdominal ultrasound can be an effective tool to make early the diagnosis of giant hydronephrosis and to perform a US guided drainage of pelvicalyceal system before open surgery. Ultrasound scan is also the first investigation mean used in detecting asymptomatic pelvicalyceal dilatation. On ultrasound scan hydronephrosis is characterised by the presence of an important anechogenic dilatation of the pelvicalyceal cavities with non-dilated ureters. The investigation of the contralateral kidney is also important to rule out an eventual bilateral hydronephrosis and/or an associated lesion in a contralateral kidney. Apart of positive diagnosis, ultrasound could lead to aetiological diagnosis in showing a urinary calculi in the ureteropelvic junction appearing as hyper echogenic, a polar vessel crossing the junction in colour Doppler US. Uro CT scan is the key tool of investigation in case of giant hydronephrosis. CT scan can evaluate the importance of the hydronephrosis, indicating the starting point in the kidney and evoking the aetiology. It can help to assess the value of the contralateral kidney and to foresee some operative difficulties. In our two cases, it is the CT Scan that help in the diagnosis and the aetiology was suspected intraoperatively despite the lack of histopathological analysis. Ureteropelvic junction syndrome is the major aetiology of giant hydronephrosis in the literature and our two cases reports illustrated it. The second main cause is represented by urolithiasis with obstructive stones (20\%) followed by congenital malformations [1]. Others causes like ureteral stenosis, retro-peritoneal fibrosis and trauma are found in the literature according to the study of Elmessaoudi and colleagues [15]. On therapeutic aspect, giant hydronephrosis could be so voluminous with important di- 
latation of pelvicalyceal system with impairment of kidney function. In these cases, nephrectomy remain the only solution of management when the contralateral kidney functioning value is normal. We realised simple nephrectomy for our two patients based on the fact that the CT scan showed complete destruction of the kidneys without secretion with any possibility to perform pyeloplasty. Urinary drainage through nephrostomy with antibiotics administration could be done while waiting for eventual nephrectomy or conservative surgery based on the renal function. Nephrostomy followed by pyeloplasty has been realised in $33 \%$ of cases in Diallo Y et coll observations at Thiès Hospital and one nephrectomy after failure of pyeloplasty were be performed in Diao's observations [7] [16].

\section{Conclusion}

Giant hydronephrosis is a rare condition of which the diagnosis is easily done by CT scan. The main aetiology has been the pyelocalyceal junction syndrome. Symptoms are poor and dominated by progressive abdominal distension leading to distorted abdomen. The ultimate evolution is destruction of renal parenchyma a non-reactive kidney. The diagnosis is done in late stage in our work environment hence the realisation of nephrectomy remains the only mean for treatment.

\section{Consent}

Consents of the children parents were obtained before publication of this article.

\section{Conflict of Interest}

The authors declared that there is no conflict of interests regarding the publication of this paper.

\section{References}

[1] Crooks, K.K., Hendren, W.H. and Pfister, R.C. (1979) Giant Hydronephrosis in Children. Journal of Pediatric Surgery, 14, 844-850. https://doi.org/10.1016/S0022-3468(79)80278-X

[2] Yang, W., Shen, S. and Wu, C. (1958) Hydronephrosis and Giant Hydronephrosis. Chinese Medical Journal, 77, 257.

[3] Kaura, K.S., Kumar, M., Sokhal, A.K., Gupta, A.K., Purkait, B., Saini, D., et al. (2017) Giant hydronephrosis: Still a Reality! Turkish Journal of Urology, 43, 337-344. https://doi.org/10.5152/tud.2017.78379

[4] Onishi, S., Nishimoto, K., Ueda, H., Okada, S., Takasaki, N. and Kaneda, K. (1985) A Case of Giant Hydro Nephrosis and a Review of 324 Cases in the Literature. Hinyokika Kiyo, 31, 129-134.

[5] Chiang, P.H., Chen, M.T., Chou, Y.H., Chiang, C.P., Huang, C.H. and Chien, C.H. (1990) Giant Hydronephrosis: Report of 4 Cases with Review of the Literature. Journal of the Formosan Medical Association, 89, 811-817.

[6] Ochsner, M.G., Fuselier, H.A., Brannan, W. and Simo, J.B. (1977) Congenital Giant Hydronephrosis in Adults. Urology, 10, 422-424.

https://doi.org/10.1016/0090-4295(77)90126-1 
[7] Diao, B., Fall, P.A., Ngom, G., et al. (2008) Giant Hydronephrosis in Child. Diagnosis and Therapeutic Features. African Journal of Urology, 14, 168-173.

[8] Hellenthal, N.J., Thomas, S.A. and Low, R.K. (2009) Rapid Onset Renal Deterioration in An Adult with Silent Ureteropelvic Junction Obstruction. Indian Journal of Urology, 25, 132-133. https://doi.org/10.4103/0970-1591.45553

[9] Vishwanath, M.P., Swamy, M.K., Neeli, S.I., Godhi, A.S. and Metgud, S.C. (2010) Giant Hydronephrosis. Indian Journal of Surgery, 72, 359-360. https://doi.org/10.1007/s12262-010-0110-8

[10] Benchekroun, A., Alami, M., Ghadouane, M., Zanoud, M., Nouini, Y., Benslimane, L., Belahnech, Z. and Faik, M. (2003) Giant Hydronephrosis. About Two Cases. Annales D'Urologie, 37, 61-64. https://doi.org/10.1016/S0003-4401(03)00004-4

[11] Kimura, R., Koyama, K. and Abe, H. (2012) A Case of Sarcomatoid Renal Cell Carcinoma Associated with Giant Hydronephrosis. Hinyokika Kiyo, 58, 435-438.

[12] Baka, K.H., Guedira, H., Lakmichi, M.A. and Dahami, Z. (2016) Hydronephrosis Revealing a Mucinous Cystadenoma of the Upper Urinary Tract. African Journal of Urology, 22, 256-258. https://doi.org/10.1016/j.afju.2016.05.003

[13] Singh, N.K., Jha, B., Khanna, R. and Khanna, N.N. (1993) Giant Hydronephrosis Masquerading as Massive Ascites. Postgraduate Medical Journal, 69, 800-802. https://doi.org/10.1136/pgmj.69.816.800

[14] Yassine, R. (2014) Pelvic Ectopic Kidney with Giant Hydronephrosis Revealed by Occlusion. African Journal of Urology, 20, 211-214.

https://doi.org/10.1016/j.afju.2014.04.004

[15] Elmessaoudi, Y.A., Rabiir, B.H., Fekak, H., Joual, A., Benani, S. and Meziane, F. (2006) Giant Hydronephrosis with Urosepsis. About an Observation and Literature Review. Journal Marocain d'Urologie, 3, 19-21.

[16] Diallo, Y., Amadou, A., Kouka, S.C., Ze Ondo, C., Sow, Y., Fall, B., Diao, B., et al. (2014) Giant Hydronephrosis. About 3 Observations and Literature Review. Journal Africain d'Imagerie Médicale, 1, 86-91. 